Ann. Biol. anim. Bioch. Biophys., I970, 10 (4), 703-706.

\title{
ÉTUDE COMPARÉE DE L'UTILISATION ÉNERGÉTIQUE DE RÉGIMES RICHES EN ACIDE CAPRYLIQUE OU EN ACIDES GRAS INSATURÉS
}

\author{
B. AURoUsseaU, L. de GROOT et M. VERMOREL \\ Station d'Étude des Métabolismes, \\ Centre de Recherches de Clermont-Ferrand, 63-Saint-Genès-Champanelle \\ Institut national de la Recherche agronomique
}

Dans le cadre des études sur la nutrition des jeunes animaux en croissance, nous nous sommes intéressés au problème des équilibres entre les diverses sources d'énergie les plus favorables à la protéinogenèse. Selon Harkins et Sarret, I968, Bollinger et Reiser, 1965, les acides gras à chaîne moyenne (l'acide caprylique et l'acide laurique de l'huile de coprah) et les acides gras longs insaturés (l'acide oléique de l'huile d'olive ou pur) semblent favoriser la croissance chez le rat. Cependant, aucune étude, à notre connaissance, n'a permis de quantifier le phénomène et de déterminer l'utilisation énergétique de ces acides gras.

Aussi avons-nous comparé l'acide caprylique sous forme de tricapryline, aux acides oléique et linoléique apportés par de l'huile d'arachide au cours d'une expérimentation en lots sur rats en croissance recevant des régimes équilibrés. Les bilans énergétiques et azotés ont été obtenus par abattage des rats et analyse des carcasses. La teneur en lipides des carcasses, leur composition en acides gras ainsi que celle des dépôts adipeux ont été déterminées, ce qui a permis de suivre les orientations du métabolisme lipidique (de Groot, Aurousseau, Vermorel, 1970).

De jeunes rats mâles Wistar sevrés vers $50 \mathrm{~g}$ ont été répartis en 3 lots comparables de 15 animaux, d'un poids vif moyen de $60 \mathrm{~g}$. Les animaux du premier lot (lot "Témoin ") ont été abattus, ceux des deux autres lots (lot "Tricapryline " et lot " Arachide 》) ont été maintenus en expérience pendant 26 jours. Les 2 régimes expérimentaux, à base d'amidon, de saccharose et de farine de hareng de Norvège ont été équilibrés au niveau de $6,2 \mathrm{mg}$ d'azote digestible (Nd) par kcal d'Énergie métabolisable (EM). La tricapryline apportait zo p. xoo de l'énergie dans l'un des régimes, l'huile d'arachide $20 \mathrm{p}$. Ioo dans l'autre. Après abattage, les carcasses ont été broyées et analysées individuellement selon la méthode décrite précédemment (VERMOREL, I968) ; les dosages de matière sèche, d'azote et d'énergie ont été effectués en triple.

Pour compléter l'interprétation de cette expérience, nous avons fait appel aux résultats obtenus avec un lot d'animaux de même âge (lot " glucides ») qui ont reçu un régime à base de glucides de même niveau azoté ( $6,2 \mathrm{mg} \mathrm{Nd} / \mathrm{kcal} \mathrm{EM})$ et ont ingéré des quantités d'énergie métabolisable voisines de celles ingérées par les deux lots expérimentaux (VERMOREL, I970).

Dans chacun des trois régimes, 2 p. roo d'huile de maïs assuraient un apport minimum d'acides gras essentiels. 
Un essai parallèle portant sur quatre animaux de chaque lot placés en cage à bilan pendant une période de $\mathbf{I} 3$ jours, a permis de déterminer, respectivement pour les régimes "Arachide " et "Tricapryline ", les coefficients de digestibilité de l'azote $(88,7$ et 89, I) et de l'énergie $(95, \mathbf{I}$ et 95,4) ainsi que les pourcentages d'énergie métabolisable de chaque régime (9I,5 et 9I,9) (tabl. I, A).

Les gains de poids vif moyens et les quantités d'azote fixées sont du même ordre dans les deux lots (tabl. I, B). La quantité d'énergie fixée par les rats du lot "Tricapryline " est inférieure de 8 p. Ioo (différence hautement significative) à celle du lot "Arachide ", correction faite pour la différence d'énergie métabolisable ingérée.

En vue de comparer l'utilisation énergétique des acides gras à celle de l'amidon, on peut calculer les quantités de EM qui auraient été nécessaires aux rats du lot "Glucides " pour fixer des quantités d'énergie identiques à celles fixées par les lots expérimentaux (tabl. 2). Le calcul montre que 210,5 et $250 \mathrm{kcal}$ EM provenant de l'amidon sont équivalentes respectivement à $24 \mathrm{I}, 5 \mathrm{kcal} \mathrm{EM}$ d'acide caprylique et $243 \mathrm{kcal}$ EM des acides gras de l'huile d'arachide.

TABLEAU I

Caractéristiques des régimes, principaux résultats expérimentaux ${ }^{1}$

\begin{tabular}{|c|c|c|c|}
\hline & $\begin{array}{c}\text { Régime } \\
\text { "Tricapryline " }\end{array}$ & $\begin{array}{c}\text { Régime } \\
\text { "Arachide" }\end{array}$ & $\begin{array}{l}\text { Régime } \\
\text { "Glucides " }\left({ }^{2}\right)\end{array}$ \\
\hline 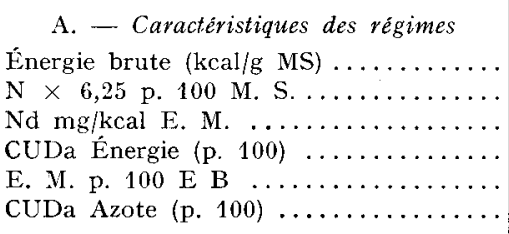 & $\begin{array}{c}4,68 \\
18,6 \\
6,1 \\
95,4 \pm 0,1 \\
91,9 \pm 0,1 \\
89,1 \pm 0,5\end{array}$ & $\begin{array}{c}4,72 \\
18,9 \\
6,2 \\
95,1 \pm 0,1 \\
91,5 \pm 0,1 \\
88,7 \pm 0,3\end{array}$ & $\begin{array}{c}4,16 \\
16,8 \\
6,2 \\
95,1 \\
91,4 \\
88,2\end{array}$ \\
\hline 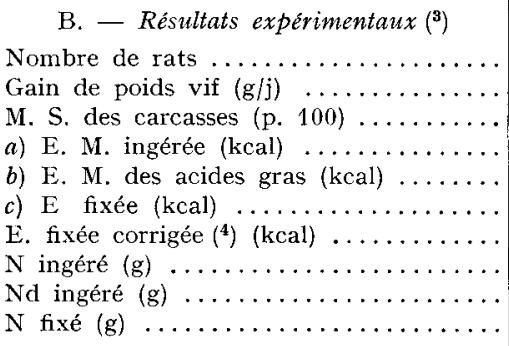 & $\begin{array}{c}14 \\
5,1 \pm 0,1 \\
33,9 * \pm 0,3 \\
1340 \pm 6 \\
241,5 \\
318 \pm 6 \\
309 * * \pm 6 \\
9,23 \pm 0,04 \\
8,22 \pm 0,04 \\
3,99 \pm 0,04(5)\end{array}$ & $\begin{array}{c}12 \\
5,2 \pm 0,2 \\
3 /, 7^{*} \pm 0,3 \\
1327 \pm 8 \\
\quad 243 \\
336 \pm 5 \\
336^{* *} \pm 5 \\
9,29 \pm 0,05 \\
8,22 \pm 0,05 \\
3,93 \pm 0,04\left({ }^{5}\right)\end{array}$ & $\begin{array}{c}15 \\
4,5 \pm 0,1 \\
36,9 \pm 0,1 \\
1320 \pm 4 \\
\pm \\
326 \\
331 \\
9,08 \pm 0,03 \\
8,01 \pm 0,03 \\
3,63\left(^{5}\right)\end{array}$ \\
\hline
\end{tabular}

(1) Valeurs moyennes et erreurs types des moyennes.

(2) Vermorel, 1970.

(3) Pour 26 jours d'expérience.

( $\left.{ }^{4}\right)$ Correction pour la différence d'E. M. ingérée, le rendement de l'utilisation de l'énergie métabolisable pour la croissance étant estimé à 71 p. 100.

$\left(^{5}\right)$ Différences significatives au seuil $\mathrm{P}=0,001$ entre le régime "glutcides " d'une part et chacun des deux lots expérimentaux.

* Différence significative au seuil $\mathrm{P}=0,02$.

** Différence significative au seuil $P=0,001$.

Donc, pour l'ensemble des fonctions, entretien et production, l'efficacité de l'utilisation de l'énergie des acides gras alimentaires par rapport à l'amidon est de 87 p. roo pour l'acide caprylique et I03 p. Ioo pour les acides gras de l'huile d'arachide. 
Le rendement inférieur de l'énergie de l'acide caprylique peut s'expliquer en partie par le métabolisme intermédiaire de cet acide. Ce dernier est dégradé en éléments à deux carbones qui sont utilisés ensuite comme source d'énergie ou incorporés dans les molécules d'acides gras longs.

La différence entre les quantités d'énergie fixée par chacun des deux lots s'explique par une différence au niveau des lipides fixés : les rats du lot "Arachide " ont déposé significativement plus de lipides que les rats du lot "Tricapryline " $(2 \mathrm{r}, 3 \mathrm{~g}$ contre $\mathrm{rg}, \mathbf{I} \mathrm{g})$ au cours de la période expérimentale.

Cet engraissement inférieur des rats du lot "Tricapryline " s'accompagne d'une teneur en matière sèche des carcasses significativement plus faible.

Aucune différence significative n'apparaît entre les quantités de protéines synthétisées par les rats des deux lots expérimentaux. En revanche, la rétention azotée est accrue significativement par l'apport d'huile d'arachide $(7,7 \mathrm{p}$. I0o) et de tricapryline $(9,0 \mathrm{p}$. I oo) en substitution de l'amidon. Toutefois, les animaux du lot "Glucides " ont ingéré 2,5 p. Ioo de moins d'azote que ceux des lots expérimentaux ; ces observations méritent d'être précisées par une nouvelle étude.

TABLEAU 2

Efficacité de l'utilisation de l'énergie des acides gras alimentaires par rapport à l'amidon

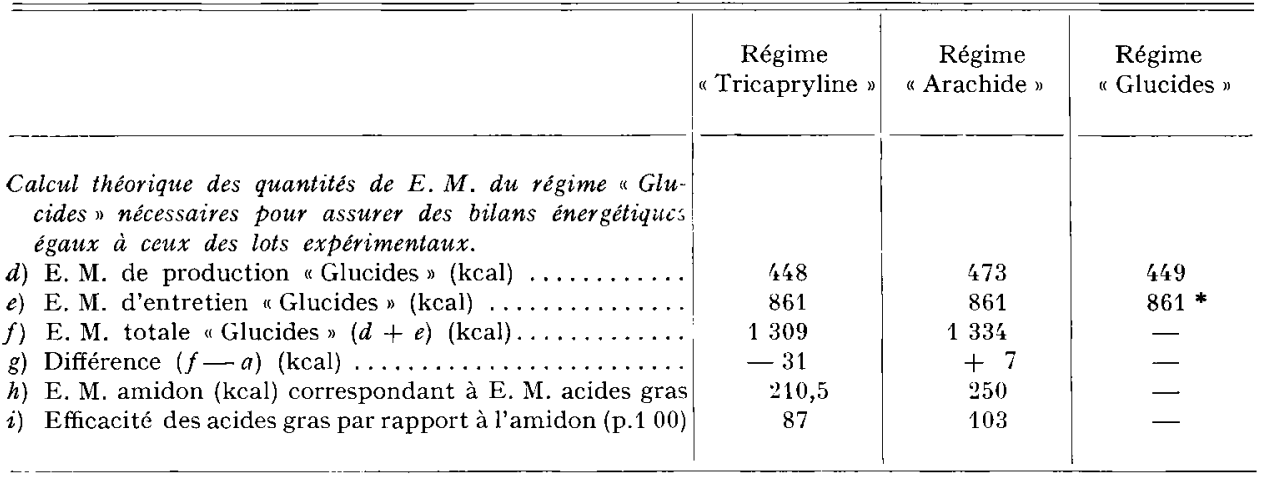

d) Besoins de production calculés en estimant à 71 p. 100 le rendement de E. M. pour la croissance (Vermorel et KEller, 1967). Les quantités d'énergie fixée (c) sont tirées du tableau 1 (B).

* Besoins d'entretien calculés par différence entre E. M. ingérée et E. M. de production. Les performances des 3 lots étant suffisamment voisines, on peut admettre que leurs besoins d'entretien sont égaux.

g) Quantité de E. M. ingérée par les animaux expérimentaux (Cf. tableau 1 B $a$ ).

$h$, i) Quantités de E. M. ingérée sous forme d'acides gras (Cf. tableau $1 \mathrm{~B} b$ ).

Ce premier travail et l'étude complémentaire sur le métabolisme lipidique (de Groor, AurousSEAU, VERMOREL, I970) confirment le fait que les acides gras longs présentent le double intérêt de se déposer directement avec un rendement énergétique élevé et de réduire les synthèses d'acides gras de novo. L'acide caprylique, pour sa part, réduit les dépôts de lipides (par suite d'une mauvaise utilisation énergétique, et réduit la proportion d'acides insaturés des dépôts. En outre, l'huile d'arachide et la tricapryline semblent favoriser les synthèses protéiques.

Ces travaux sont actuellement poursuivis par l'étude de l'utilisation par le rat en croissance de régimes comportant différents acides gras apportés en substitution à une partie de l'amidon du régime de base. L'emploi d'éléments marqués au ${ }^{14} \mathrm{C}$ permettra de préciser la participation de ces acides gras aux synthèses lipidiques. 


\section{SUMMARY}

\section{COMPARISON OF ENERGY UTILIZATION OF DIETS WITH HIGH LEVELS OF CAPRYLIC ACID. V. - UNSATURATED FATTY ACIDS}

Two diets with 20 p. Ioo utilizable energy either as tricaprylin or as groundnut oil, were given to Wistar growing rats. Energy and nitrogen balance were estimated from the analysis of carcasses.

At day 26 of the experiment, rats given the tricaprylic diet had 8 p. roo less energy than those given the groundnut diet $(\mathrm{P}=0.00 \mathrm{I})$. The utilization of metabolizable energy compared to that of starch was $103 \mathrm{p}$. I oo for groundnut oil fatty acids v. 87 p. roo for caprylic acid.

Compared to a control diet without fatty acids, protein synthesis was $7.7 \mathrm{p}$. Ioo higher with groundnut oil and $9.0 \mathrm{P}$. I 00 higher with tricaprylin $(\mathrm{P}=0.00 \mathrm{I})$.

\section{RÉFÉRENCES BIBLIOGRAPHIQUES}

Harkins R. W., Sarret H. P., ig68. Nutritional Evaluation of Medium chain Triglycerides in the rat. J.A.O.C.S., 45, 26-30.

Bollinger J. N., Reiser R., I965. The metabolic fate of fatty acids derived from dietary Triglycerides. J.A.O.C.S., 42, II30-II33.

De Groot I., Aurousseau B., Vermorel M., ig7o. Influence de l'ingestion d'acide caprylique et d'acide oléique sur le métabolisme lipidique du rat en croissance. C. R. Acad. Sci., 270, 721 .

Vermorel M., 1968. Utilisation énergétique de la triacétine par le rat en croissance. Ann. Biol. anim. Bioch. Biophys, , 8, 453-455.

Vermorel M., I97o. Utilisation énergétique du diacétate de propylène glycol par le rát en croissance. Ann. Biol. anim. Bioch. Biophys., 10,33I-333.

Vermorel M., Keller J., I 967 . Utilisation énergétique par le rat en croissance, des principales céréales composant des régimes isoazotés et équilibrés en acides aminés. Ann. Zootech., 16, 223-234. 